\title{
Special Mitral Valve Issue
}

Beginning with Da Vinci, the structure and function of the mitral valve have been of great interest to anatomists, physicians, surgeons, scientists, and engineers. Yet, a complete understanding of the complex mechanical function of the normal mitral valve, as well as the biological behavior of the native valve tissue in both health and disease remains elusive. However, much progress has been made, particularly in recent years. This special issue of CVET presents some of the latest scientific studies that address normal function, changes due to disease or disruption, and surgical approaches for repair and replacement.

Initially, the surgical approach to address mitral valve disease was "simple" valve replacement beginning in the early 1960 s, in which the native valve structures were removed, and a prosthetic valve sewn in. The first commercially available valves were ball and cage designs, tilting disk, and bi-leaflet valves, none of which are anatomically similar to the native mitral structure. Even though design improvements led to superior hemodynamics, it was shown that simply replacing the open/close function of the leaflets, without preservation of the subvalvular apparatus, led to suboptimal results.

Dr. Carpentier changed the surgical landscape in 1983 with his publication of the landmark article "the French Correction". He presented new surgical repair techniques, with the goal of returning the valve to a normal anatomical, and thus functional, state without the need for replacement. During the eighties, continuing work by Carpentier, Duran, Frater, and David convincingly demonstrated the superiority of mitral valve repair. Now, practicing surgeons can perform mitral repair in more than $90 \%$ of cases in many centers.

Still, many questions remain to be addressed in regard to structure, function, and optimization of repair for individual patients. With the advent of computer aided technology, engineers and scientists working together with surgeons began to address the goal of patient specific modeling in the early 1990s. The early finite element models provided insight into the necessity for restoration of the native anatomical structure during surgical repair, to achieve normal mechanical function. It is only recently that improvements in imaging technology have allowed reconstruction of truly patient specific models including all relevant anatomy, including the left ventricle.
In this special issue, three articles present some of the most recent advances in computational modeling. Domenichini presents an asymptotic model of fluidtissue interaction to evaluate intraventricular fluid dynamics and the vortex formation in their relationship with mitral valve motion. Guccione's group presents a fully 3-D mechanical finite element model of the mitral valve integrated with the complete anatomy of the heart, and applies it to evaluate a novel annuloplasty ring for ischemic MR. And lastly, Votta's group presents a finite element mechanical model, developed from patient specific images, to evaluate edge-to-edge repair. Ultimately the integration and combination of these types of models will be able to provide us with whole heart analysis, and patient-specific surgical planning.

However, another road block has been the lack of detailed knowledge with regard to biological and material properties, and how the native tissue may remodel itself in response to normal or abnormal conditions. Grande's group has led the way in this area, and in conjunction with Miller's group, presents two relevant articles. The first addresses mitral leaflet matrix composition and changes related to tissue strain, and the second addresses how the cellular and matrix composition affects annular contraction. This area of work is necessary not only to improve computational models as they increase in complexity - but also to be able to potentially identify cellular or biologic therapeutic targets for addressing mitral valve disease in situ, and thus potentially avoiding surgery altogether.

In addition to improvements in technology and biology for basic science assessment, there have been concurrent clinical advances, particularly with regard to minimally invasive and robotic approaches. Minimally invasive procedures have now become the standard approach in many centers. However, questions remain as to whether the outcomes are as favorable, compared to conventional mitral valve surgery through a median sternotomy. Falk's group summarizes the current literature comparing minimally invasive mitral valve surgery and conventional mitral valve surgery. Del Nido's group presents an integrated approach for treatment of congenital mitral stenosis including pre-operative imaging, aggressive surgical techniques and peri-operative management.

While the superiority of mitral valve repair is universally accepted, there will always remain a subgroup 
of patients in which repair is not possible. For such patients, there have been significant improvements in surgical technique, and surgical approaches to mitral valve replacement. Burriesci provides us with a review of minimally invasive mitral valve replacements, particularly those devices currently at a most advanced stage of development.

As new devices and percutaneous approaches continue to be developed, rapid preclinical evaluation of the function and mechanics can still best be accomplished by bench models of the left ventricle. Siefert's review aims to provide a comprehensive assessment of these models to aid interpretation of their resulting data, inform future experimental evaluations, and further the translation of results to procedure and device development.

As yet, we still do not have the "perfect" answer for addressing abnormal mitral valve physiology. However, all of the advances described in this special issue will contribute toward reduced operative time, less invasive and less traumatic surgical techniques, and improved physiologic outcomes, and quicker recovery for the patients. We still have many things to learn in regard to native valve structure and function, changes in the disease process, choosing the optimal timing for intervention, and utilizing the best materials for repair or replacement. However, it is the collaborative work between scientists, engineers, and clinicians that will continue to answer important questions, and perhaps ultimately lead us to a "personalized medicine" approach for effective treatment of mitral valve disease, through the integration of comprehensive computational modeling, biological tissue function evaluation, and implementation of novel clinical procedures. I hope that you enjoy this selection of articles, and come away with a new understanding of some of the most recent research in this area.

KARYN S. KUNZELMAN

Department of Mechanical Engineering

University of Maine,

219 Boardman Hall,

Orono, ME, USA

Electronic mail: kunzelka@msn.com,

karyn.cochran@maine.edu 